\title{
Anomalous backscattering and absorption in second harmonic ECRH experiments due to low-threshold two UH plasmon decay instability in blobs
}

\author{
Evgeniy Gusakov*, Aleksei Popov, and Ekatherine Sysoeva \\ Ioffe Institute, 194021 St-Petersburg, Russia
}

\begin{abstract}
An universal mechanism responsible for excitation of the low-threshold two upper-hybrid (UH) plasmon absolute parametric decay instability of a pump extraordinary microwave is proposed and analysed in detail. A corner-stone of it is the 2D localization of the daughter UH waves in an intensive magnetic field aligned blob/filament and the presence of a finite-width mm-wave pump beam
\end{abstract}

\section{Introduction}

In the recent decade a substantial number of observations in the electron cyclotron resonance heating (ECRH) experiments in tokamaks and stellarators had been made which do not fit into a simple linear picture. The most eloquent among them is the anomalous backscattering phenomena studied in detail in the second harmonic X-mode heating experiments on TEXTOR $[1,2]$. The theoretical model [3] proposed recently explains this anomalous backscattering as a result of the two upper-hybrid (UH) plasmon parametric decay instability (PDI) possessing very low threshold due to trapping of nonlinearly excited plasmons in the vicinity of the density maximum that accompanies the magnetic island [4]. The theoretical model [3] also predicts substantial (up to $25 \%$ ) anomalous absorption due to this process. This makes important further detailed analysis of the mechanisms and scenarios responsible for excitation of the low-power-threshold PDI of an extraordinary wave.

The density fluctuations can also lead to anomalous absorption of mm-wave [5]. In particular, the large amplitude density fluctuations routinely observed in experiments at the plasma edge [6-8] in the form of blobs or filaments, aligned with the magnetic field line and possessing the finite size perpendicular to it can ensure the 2D localization of the daughter UH waves and, thus, serve as potential centers of low-threshold two-plasmon parametric decay and sources of anomalous scattering of mm-waves.

In the present paper we analyze the scenario of the low-threshold PDI associated with excitation of UH waves $2 \mathrm{D}$ trapped in plasma blobs which is applicable to both the stellarator and tokamak second harmonic ECRH experiments. The numerical estimations for this scenario are given in the paper for conditions of the second harmonic ECRH experiment in ASDEX Upgrade [9].

\section{Parametric excitation of the 2D trapped UH waves by the pump extraordinary wave}

In order to describe nonlinear excitation of the UH waves trapped in a blob aligned with the magnetic field line, we introduce the local cylindrical and Cartesian coordinate systems $(r, \theta, z)$ and $(x, y, z)$ with their common origin located in the centre of a blob and the coordinate $z$ directed along the magnetic field line. The coordinate $x$ is related to the flux surface label, and $y$ is the coordinate perpendicular to the magnetic field line on the magnetic surface. We focus on the ECRH experiments being carried out in ASDEX Upgrade [9] and model the density profile as a sum of the background smoothly inhomogeneous term $n_{0}$ and a sharp perturbation arising due to the presence of a blob generated by a low-frequency instability of MHD or drift type and given in the local cylindrical coordinate systems $(r, \theta, z)$ by expression

$\delta n=\delta_{n} n_{0} \exp \left(-r^{2} / l_{b}^{2}\right)$

In the above formula $\delta_{n}<<1$ is the relative amplitude of a density perturbation and $l_{b}$ is the width of it. Thus, the density perturbation (1) models a structure being aligned with the magnetic field line and possessing the finite size perpendicular to it. For our numerical analysis we choose its width in the millimeter scale range $l_{b}=0.3 \mathrm{~cm}$ corresponding to the trapped electron mode typical for the ECRH experiments. The perturbation relative amplitude used in the analysis $\delta_{n}=0.25$ is consistent with the values usually observed at the edge plasma [8]. We assume temporal variation of the density fluctuation negligible during the period or inverse growth rate of the decay waves under consideration in this paper. 
Then, we analyze the three-wave interaction model relevant to the experiment. We assume that the pump extraordinary wave propagates almost perpendicular to the magnetic field in the density inhomogeneity direction with its dominant polarization vector component being mostly directed along the coordinate $y$. We represent the X-mode pump propagating through the decay layer as

$\mathbf{E}=\mathbf{e}_{y} \frac{E_{0}}{2} \exp \left(i k_{x} x-i \omega_{0} t\right)+c . c$.

where $E_{0}(y, z) \equiv \sqrt{8 P_{0} /\left(c w^{2}\right)} \exp \left(-\left(y^{2}+z^{2}\right) / 2 w^{2}\right)$ is the wave amplitude with $P_{0}$ and $w$ representing the pump power and the width of a beam, $k_{x}\left(\omega_{0}\right) \square \omega_{0} / c$ being a component of the wavenumber is nearly constant for the typical conditions of the $2^{\text {nd }}$ harmonic ECRH experiments where the inequalities $\omega_{0}^{2} \gg>\omega_{c e}^{2}, \omega_{p e}^{2}$ usually hold, and c.c. is the term derived from the first one by complex conjugation. In the cylindrical coordinate system (2) reads

$\mathbf{E}=\frac{E_{0}(z)}{2}\left[\mathbf{e}_{r} \sin \theta+\mathbf{e}_{\theta} \cos \theta\right] \times$

$\sum_{m=-\infty}^{\infty} J_{m}\left(k_{x} r\right) \exp \left(i m \theta-i \omega_{0} t\right)+c . c$.

where we have used the inequality $l_{b}<<w$ and introduced Bessel function of the first kind $J_{m}$.

We consider the pump wave decay into a couple of the electrostatic UH waves propagating in opposite directions and represented by the potentials $\varphi_{1,2}(\mathbf{r}, t)=\phi_{1,2}(\mathbf{r}) \exp \left(\mp i \omega_{1,2} t\right) / 2+$ c.c.,$\quad \omega_{2}=\omega_{0}-\omega_{1} . \quad \mathrm{n}$ weakly inhomogeneous plasma the set of equations describing this nonlinear coupling reads

$\hat{D}_{1} \phi_{1}(\mathbf{r})=4 \pi \rho_{1}$,

$\hat{D}_{2} \phi_{2}(\mathbf{r})=4 \pi \rho_{2}$

where

$\hat{D}_{1,2} \equiv \hat{D}\left(\omega_{1,2}\right)=l_{T 1,2}^{2}\left(\frac{1}{r} \frac{\partial}{\partial r} r \Delta_{\perp} \frac{\partial}{\partial r}+\frac{1}{r} \Delta_{\perp} \frac{1}{r} \frac{\partial^{2}}{\partial \theta^{2}}\right)-\varepsilon \Delta_{\perp}^{2}-$

$\frac{\partial \varepsilon}{\partial r} \frac{\partial}{\partial r}-\eta \frac{\partial^{2}}{\partial z^{2}}-\frac{\omega_{1,2}^{2}}{c^{2}}\left(\varepsilon^{2}-g^{2}\right), \Delta_{\perp}=\frac{1}{r} \frac{\partial}{\partial r} r \frac{\partial}{\partial r}+\frac{1}{r^{2}} \frac{\partial^{2}}{\partial \theta^{2}}$,

$l_{T 1,2}^{2} \equiv l_{T}^{2}\left(\omega_{1,2}\right)=\frac{3}{2} \frac{v_{t e}^{2}}{4 \omega_{c e}^{2}-\omega_{1,2}^{2}}$,

$\varepsilon, g$ and $\eta$ are the components of the "cold" dielectric tensor. The nonlinear induced charge densities $\rho_{1,2}$ arising on the RHS of equations (4) describe coupling of the daughter UH waves in the presence of the pump wave (2). Their explicit representation is given below.

Then, we assume the weak nonlinear coupling of UH waves and utilize the perturbation theory approach [10]. In the first step of the perturbation procedure we put $\rho_{1,2}=0$ and analyze the homogeneous differential equations $\hat{D}_{1} \phi_{1}(\mathbf{r})=0$ and $\hat{D}_{2} \phi_{2}(\mathbf{r})=0$. We start with consideration of the 1D problem and show in figure 1 the 1D dispersion curves of the UH wave $\left(f_{1}=70 \mathrm{GHz}\right)$ in the presence of the blob (1) for conditions of the second harmonic ECRH experiments in ASDEX-Upgrade

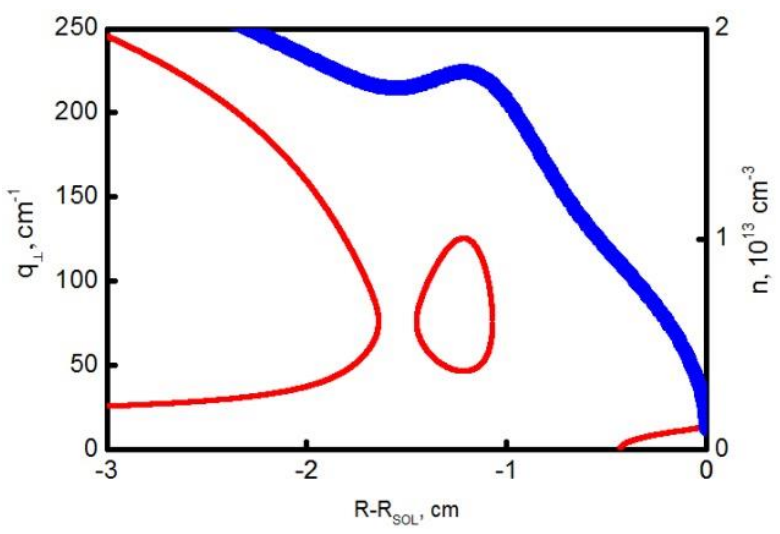

Fig. 1. (Left and bottom axes) - The 1D dispersion curves $q_{\perp}$ of the $\mathrm{UH}$ wave $\left(f_{0} / 2=70 \mathrm{GHz}\right.$ - dash-dotted curve. (Right and bottom axes) - the density profile (thick solid line).

$\left(B_{0}=3.4 \mathrm{~T},\left\langle n>=9 \times 10^{13} \mathrm{~cm}^{-3}, T_{e, e \text { edge }}=150 \div 200 \mathrm{eV}\right.\right.$, the system operates at $140 \mathrm{GHz}$ with $\mathrm{X} 2$ scheme) [9]. The chosen edge density profile is also depicted in the figure 1 in thick solid curve. As we can see, the UH wave can be localized in a vicinity of the local maximum of the density profile. Then, we get back to the realistic 2D problem and demonstrate the possibility of 2D localization of both the UH waves in the blob. Seeking solutions of the homogeneous equations by means of the WKB approximation and assuming the high azimuthal modes $n_{1,2}>1$ of the UH waves we get

$$
\begin{aligned}
& \phi_{1,2}(\mathbf{r})=C_{1,2} F_{1,2}, \\
& F_{1,2}=\frac{1}{2 \sqrt{q_{1,2 r}(r)}} \exp \left(i \int^{r} q_{1,2 r}(\xi) d \xi+i n_{1,2} \theta+i q_{z} z\right)+c . c .
\end{aligned}
$$

where

$$
q_{1,2 r}=\frac{1}{l_{T 1,2}} \sqrt{-\frac{\varepsilon}{2 l_{T 1,2}^{2}}-\sqrt{\frac{\varepsilon^{2}}{4 l_{T 1,2}^{4}+\left(\frac{\omega_{1,2}^{2} g^{2}}{c^{2} l_{T 1,2}^{2}}+\frac{q_{z}^{2} \eta}{l_{T 1,2}^{2}}\right)}}-\frac{n_{1,2}^{2}}{r^{2}}} .
$$

In the absence of the nonlinear pump the amplitudes of the $\mathrm{UH}$ waves are constant, $C_{1,2}=$ const. The frequencies $\omega_{1}^{k, l}, \omega_{2}^{k, l}=\omega_{0}-\omega_{1}^{k, l}$ and wave-number $q_{z}^{k, l}$ of them obey simultaneously the Bohr-Zommerfeld quantization conditions

$$
\begin{aligned}
& \int_{r_{r l}}^{r_{r}^{*}} q_{r 1}\left(\xi, \omega_{1}^{k, l}, q_{z}^{k, l}\right) d \xi=\pi(k+1 / 2), \\
& \int_{r_{2, l}}^{r_{r}^{*}} q_{r 2}\left(\xi, \omega_{0}-\omega_{1}^{k, l}, q_{z}^{k, l}\right) d \xi=\pi(l+1 / 2) .
\end{aligned}
$$

The two pairs of the coordinates $\left(r_{1 l}^{*}, r_{1 r}^{*}\right)$ and $\left(r_{2 l}^{*}, r_{2 r}^{*}\right)$ in (5) are the solutions of two equations $q_{1 r}\left(r_{1, r}^{*}\right)=0$ and $q_{2 r}\left(r_{2 l, r}^{*}\right)=0$, corresponding to the $\mathrm{UH}$ wave turning points in the radial direction. For the conditions used the decay of the azimuthal modes $m= \pm 1$ (see equation (3)) forming the symmetric pump

$$
\mathbf{E}^{m=1}=\mathbf{e}_{r} \frac{E_{0}(z)}{2} J_{1}\left(k_{x} r\right) \exp \left(i \frac{\pi}{2}-i \omega_{0} t\right)+c . c .
$$

into the $\mathrm{UH}$ waves with the equal frequency $f_{1}=f_{2}=f_{0} / 2=70 \mathrm{GHz}$ and the poloidial mode numbers $n_{1}=n_{2}=n=13 \quad\left(q_{z}=0.37 \mathrm{~cm}^{-1}\right)$ seems to be the most dangerous because the decay condition for radial wavenumbers in this case is satisfied all over the 


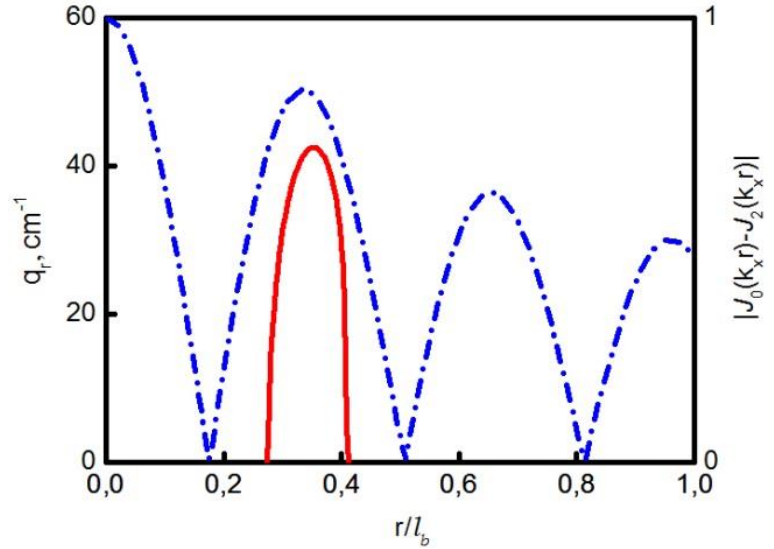

Fig. 2. (Left and bottom axes) - Radial wavenumber $q_{r}$ of the $\mathrm{UH}$ waves $\left(f_{1}=70 \mathrm{GHz}, n_{1}=13\right)$ versus the dimensionless blob radius. (Right and bottom axes) the radial profile of $\left|J_{0}\left(k_{x} r\right)-J_{2}\left(k_{x} r\right)\right|$ (dash-dotted line).

plasmon localisation region. In (6) we have omitted the terms $\propto \exp ( \pm i 2 \theta)$ which are unable to afford resonant nonlinear coupling with the selected daughter UH waves.

In order to describe the nonlinear coupling we introduce the charge densities $\rho_{1,2}$ which are given at $q_{r}<<m / r$ by the following equations

$\left[\begin{array}{c}\rho_{1} \\ \rho_{2}\end{array}\right]=\frac{|e|}{4 \pi m_{e}} \frac{n^{2}}{r^{2}} \frac{\omega_{p e}^{2} \omega_{1} \omega_{2}}{\Delta_{0} \Delta_{1} \Delta_{2}} \frac{\partial J_{1}\left(k_{x} r\right)}{\partial r}\left[\begin{array}{c}E_{0}(z) \phi_{2} \\ E_{0}^{*}(z) \phi_{1}\end{array}\right]$.

with $\Delta_{j}=\omega_{j}^{2}-\omega_{c e}^{2}, j=0,1,2$. In figure 2 we demonstrated the dispersion curves of the daughter $\mathrm{UH}$ waves corresponding to fundamental radial modes being merged along with the radial distribution of the spatial form-factor determining the efficiency of the nonlinear interaction of pump and daughter waves, $2\left|\partial J_{1}\left(k_{x} r\right) / \partial\left(k_{x} r\right)\right|=\left|J_{0}\left(k_{x} r\right)-J_{2}\left(k_{x} r\right)\right|$. As it is seen there, the daughter UH waves possessing large azimuthal number are localised in a narrow ring within the second maximum of the form-factor $2\left|\partial J_{1}\left(k_{x} r\right) / \partial\left(k_{x} r\right)\right|$. The latter makes strong nonlinear coupling possible.

In the second step of the perturbation procedure we take into account the nonlinear coupling and the daughter waves energy losses along the axis $\mathbf{e}_{z}$. The amplitudes of UH waves in this case are no longer constant, i.e. $C_{1,2} \rightarrow C_{1,2}(z, t)$. We substitute (2) into (4), multiply the first line by $F_{1}^{*}$, the second one by $F_{2}^{*}$ and integrate them over the coordinate $r$. Eventually, we arrive at

$\left\{\begin{array}{l}\frac{\partial C_{1}}{\partial t}+u_{z} \frac{\partial C_{1}}{\partial z}=v_{0} \frac{E_{0}(z)}{H} C_{2} \\ \frac{\partial C_{2}}{\partial t}-u_{z} \frac{\partial C_{2}}{\partial z}=v_{0}^{*} \frac{E_{0}^{*}(z)}{H} C_{1}\end{array}\right.$

where $u_{z}$ is the group velocity of both the UH plasmons averaged over their radial localization area and describing their convective energy losses along the axis $\mathbf{e}_{z}$. The term describing nonlinear coupling of the UH plasmons $\left(v_{0}\right)$ is defined as follows

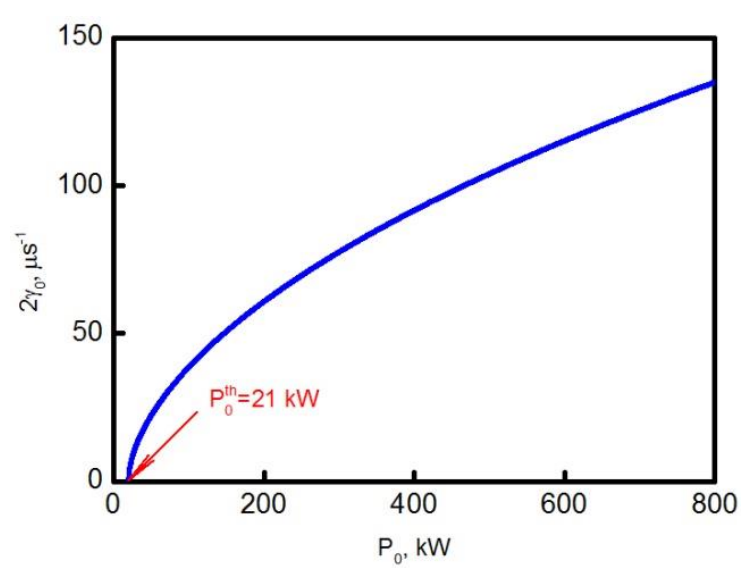

Fig. 3. The power dependence of the growth-rate $2 \gamma_{p}$

(9); $p=0$.

$v_{0}=\left.\frac{\omega_{0}\left|\omega_{c e}\right| k_{x} c}{16 \Delta_{0}}\right|_{x=x_{b}} \frac{\int_{r_{l l} \mid}^{r_{1} r} \frac{d r}{\left|q_{r 1}\right|}\left(\frac{n}{r}\right)^{2}\left(J_{0}\left(k_{x} r\right)-J_{2}\left(k_{x} r\right)\right)}{\int_{r_{l} /}^{r_{1}} \frac{d r}{\left|q_{r 1}\right|}\left(\frac{n}{r}\right)^{2}}$,

with $x_{b}$ indicating a position of the blob. For the parameters used the coefficients in (7) are $u_{z}=2.76 \mathrm{~cm} / \mu \mathrm{s}$ and $\left|v_{0}\right| E_{0}(0) / H=11 \mu \mathrm{s}^{-1}$. It should be stressed that returning back to the decay region of even a part of energy, lost from it convectively along the magnetic field is leading to the feed-back effect and as a result to a possibility of the temporal exponential growth of the daughter waves. Such a feed-back is created when in the spot of a finite-width pump beam the daughter waves propagate in opposite directions. Following [2,3] we seek the temporally growing solution of equations (7) by means of the WKB approximation, i.e. assuming

$C_{1,2} \propto q_{z}^{-1 / 2}(z) \exp \left(\gamma t+i \int^{z} q_{z}\left(z^{\prime}\right) d z^{\prime}\right)$.

This leads to the dispersion relation

$u_{z}^{2} q_{z}^{2}+\gamma^{2}=\left|v_{0}\right|^{2} \frac{E_{0}(z)^{2}}{H^{2}}$

If the pump power is large enough, we use the Taylor expansion of the pump beam distribution along the magnetic field $E_{0}^{2}(z) \approx E_{0}^{2}(0)\left(1-z^{2} / w^{2}\right)$ and get from (8) the expression for the wavenumber of the UH wave

$q_{z}=\frac{\left|v_{0}\right|}{u_{z}} \frac{E_{0}(0)}{H} \sqrt{\left(1-\frac{\gamma^{2}}{\left|v_{0}\right|^{2}} \frac{H^{2}}{E_{0}^{2}(0)}\right)-\frac{z^{2}}{w^{2}}}$.

valid at $\gamma \approx\left|v_{0}\right| E_{0}(0) / H$. Then, we utilize the quantization procedure $\int q_{z} d z=\pi(2 p+1)$ and get explicitly the growth rate of the eigen mode of the daughter UH plasmon

$\gamma_{p}=\left|v_{0}\right| \frac{E_{0}(0)}{H} \sqrt{1-(2 p+1) \frac{u_{z}}{\left|v_{0}\right| w} \frac{H}{E_{0}(0)}}$

The power dependence of the growth rate is shown in figure 3 where a surprisingly low power threshold of the decay of only $P_{0}^{\text {th }}=21 \mathrm{~kW}$ is seen. At the higher heating power $P_{0}=500 \mathrm{~kW}$ typical for the ECRH experiments in ASDEX Upgrade [9] and exceeding drastically the PDI 
power threshold the growth rate for the fundamental mode is extremely large $2 \gamma_{0} \approx 10^{8} \mathrm{~s}^{-1}$ similar to the case of two-plasmon decay in the magnetic island [3]. Based on this similarity one can expect that the decay instability will affect the ECRH power budget in the case of blob, as in [3]. This makes actual correct estimation of the amount of pump power gained anomalously by the daughter UH waves. To this end it is necessary to clarify a saturation mechanism of the decay and to evaluate its saturation level. Most likely, the PDI leading to amplification of the UH waves from the thermal noise level in a local area within the pump beam, the length of which is $\delta z_{p}=2(2 p+1) u_{z} w H /\left(\left|v_{0}\right| E_{0}(0)\right)$, is saturated due to the nonlinear effects. The most low-threshold among them is the secondary PDI driven by the daughter UH wave which gives rise to the secondary UH wave trapped in the blob and ion Bernstein wave. The nonlinear coupling of different UH plasmons, as it is shown in [3], can lead to generation of the microwaves frequency downshifted in respect to the pump. Observation of this waves by the collective Thomson Scattering (CTS) diagnostics is interpreted as strong anomalous scattering of the powerful pump wave [9].The detailed analysis of the saturation mechanism of the primary PDI of the pump wave and of the accompanying electromagnetic radiation will be presented elsewhere.

\section{Conclusions}

In the paper we analysed the possibility of the low-power-threshold two-UH-plasmon parametric decay of the pump extraordinary wave in the second harmonic ECRH experiments. The corner-stone of the proposed model is the presence of a helical coherent structures, i.e. blobs or filaments, arising in the plasma turbulence, aligned with the magnetic field line and leading to the 2D nonmonotonous plasma density profile. The UH waves are shown to be easily captured in a plane perpendicular to the magnetic field in these 2D structures. The presence of the finite-width pump beam leads to a feed-back effect for the daughter UH waves propagating along the magnetic field line in opposite directions and to their temporal exponential growth, which can be interpreted as an excitation of the absolute two-UH-plasmon PDI. We derive expressions for the instability power-threshold and its growth rate for the actual experimental plasma parameters of the second harmonic ECRH experiments in ASDEX Upgrade and the realistic filament parameters. The analyzed parametric decay possesses a very low threshold power of several tens $\mathrm{kW}$ and the growth rate being extremely large $2 \gamma_{0}=10^{8} \mathrm{~s}^{-1}$, which is similar to [3]. The saturation of this strong instability could occur at the level leading to substantial power transfere into $\mathrm{UH}$ waves affecting the power budget of the ECRH as it according to [3] was the case in ECRH experiments in TEXTOR tokamak [1, 2]. The nonlinear coupling of the UH plasmons is a possible candidate for explanation of the anomalous scattering effect observed in the ECRH experiments in ASDEX Upgrade tokamak [9].

The financial support of the RSF grant 16-12-10043 and of the Ioffe Institute is acknowledged.

\section{References}

[1] E. Westerhof, S.K. Nielsen, J.W. Oosterbeek et al. Phys. Rev. Lett. 103, 125001 (2009).

[2] S.K. Nielsen, M. Salewski, et al. Plasma Phys.

Control. Fusion 55, 115003 (2013)

[3] E.Z. Gusakov and A.Yu. Popov Physics of Plasmas 23, 082503 (2016)

[4] A.Yu. Popov, E.Z. Gusakov Plasma Phys. Control. Fusion 57, 025022 (2015)

[5] E. Gusakov, A. Popov, A., Saveliev Plasma Phys. Control. Fusion 56, 015010 (2014)

[6] S.I. Krasheninnikov Physics Letters A 283, 368370 (2001)

[7] S.J. Zweben, R.J. Maqueda, D.P. Stotler et al. Nuclear Fusion 44, 134-153 (2004)

[8] B. Vanovac, I.G.J. Classen, S.S. Denk et al. Proc. 43rd EPS Conference on Plasma Physics 40A P1.014

[9] S.K. Nielsen, W. Bongers, S. Fietz et al. Proceedings of the 9th International Workshop "Strong Microwaves and Terahertz Waves: Sources and Applications" (2014)

[10] E.Z. Gusakov, V.I. Fedorov Sov. J. Plasma Phys. 5, 463 (1979) 